\title{
Estudio contrastivo de las competencias orales en las aplicaciones móviles (apps) para el estudio del Francés Lengua Extranjera (FLE)
}

\section{Contrastive study of oral skills in mobile applications (apps) for the study of French as a Foreign Language (FFL)}

\author{
Ma José Arévalo Benito \\ Arrate Aldama Epelde \\ Universidad del País Vasco/Euskal Herriko Unibertsitatea \\ mariajose.arevalo@ehu.eus \\ arrate.aldama@ehu.eus
}

\begin{abstract}
The purpose of this article is to establish a comparative analysis of four mobile applications (apps) designed to acquire French as a Foreign Language (FFL). Two of them are aimed at a general public where French is one of the languages offered: Bussu and Duolingo. The other two were created exclusively for FLE learning: Le Bon Mot and Français Premiers Pas. The bibliographical review allows us to affirm that autonomy and simplicity are assets of the use of apps, while the repetitive side remains a constraint. We will then focus on the study of the exercises proposed in the selected apps with regard to the oral skills of comprehension and expression. Our study comes to the conclusion that, even if comprehension skills are quite well developed, expression skills could be improved to make these apps efficient tools.
\end{abstract}

\section{Key-words}

apps, contrastive study, oral skills, French as a Foreign Language.

\section{Résumé}

Le but de cet article est d'établir une analyse comparative de quatre d'applications mobiles (apps) conçues pour acquérir le Français Langue Étrangère (FLE). Deux d'entre elles sont dirigées à un public généraliste où le français est l'une des langues proposées: Bussu et Duolingo. Les deux autres ont été créées exclusivement pour l'apprentissage du FLE: Le Bon Mot et Français Premiers Pas. La révision bibliographique nous permet d'affirmer que l'autonomie et la simplicité sont des atouts de l'utilisation des apps, tandis que le côté répétitif reste une contrainte. Nous nous centrerons, par la suite, dans l'étude des exercices proposés dans les applications choisies en ce qui concerne les compétences orales de compréhension et l'expression. Notre étude arrive à la conclusion que, même si les compétences de compréhension se trouvent assez bien développées, celles d'expression pourraient améliorer pour faire de ces apps des outils performants.

Mots-clés

apps, analyse contrastive, compétences orales, FLE. 
Anales de Filología Francesa, n. ${ }^{\circ}$ 29, 2021

ESTUDIO CONTRASTIVO DE LAS COMPETENCIAS ORALES EN LAS APLICACIONES MÓVILES (APPS)...

\section{Introducción}

Desde la aparición de Internet, las TIC ocupan un lugar imprescindible en el aprendizaje de las lenguas y más concretamente en el Francés como Lengua Extranjera (FLE). A medida que la tecnología se va desarrollando, el número de dispositivos digitales aumenta y, como consecuencia, las nuevas herramientas que se pueden poner a disposición del aprendizaje de las Lenguas Extranjeras (LE) no paran de crecer.

En este artículo nos interesamos por unas herramientas relativamente recientes, las aplicaciones móviles (aquí las denominaremos apps) que se instalan en los dispositivos móviles como teléfonos o tabletas. Entre la variedad de apps concebidas para diferentes usos, encontramos en el mercado aquellas cuyo propósito es el aprendizaje de idiomas. Para realizar nuestro análisis hemos escogido cuatro aplicaciones: dos generalistas y dos concebidas específicamente para el estudio del FLE, y nos centraremos en el desarrollo de las competencias orales, tanto de expresión como de comprensión en el ámbito del FLE.

En un primer momento, estableceremos una revisión bibliográfica sobre las apps como instrumento para el desarrollo del aprendizaje de lenguas. Posteriormente, presentaremos las aplicaciones seleccionadas y pasaremos a realizar el análisis de los ejercicios propuestos para el desarrollo de la comprensión y la expresión orales, y así poder medir su potencial didáctico.

\section{Las aplicaciones móviles en el aprendizaje de una Lengua Extranjera (LE)}

Los teléfonos inteligentes o smartphones llevan en el mercado desde el 2007 (fecha del lanzamiento del primer modelo de iphone $\left.{ }^{\circledR}\right)$. Las tabletas, por su parte, empezaron a comercializarse en el año 2010. A partir de entonces su utilización se ha difundido por todo el mundo y ya resultan imprescindibles para una gran parte de la población del s. XXI.

Las aplicaciones móviles han sido desarrolladas para instalarse en dichos smartphones o tabletas. Su ámbito de uso se ha extendido a múltiples funciones respondiendo a un gran número de necesidades tanto de ocio como de consumo, salud o vida cotidiana, entre otras. Entre ellas, se da una gran oferta de apps educativas y, más concretamente, de apps destinadas al aprendizaje de lenguas que es el objeto de nuestro artículo.

Aunque relativamente recientes, estas aplicaciones han sido objeto de investigaciones que confirman sus ventajas en el aprendizaje de lenguas (Godwin-Jones, 2011; Lafford, 2011; Kim \& Kwon, 2012; Burston, 2014) cuya base teórica se enmarca en la Teoría de la Adquisición de Segundas Lenguas (Second Language Acquisition, SLA de sus siglas en inglés), el Aprendizaje de Lenguas Asistido por Ordenador (Computer Assisted Language Learning, CALL de sus siglas en inglés) y, más específicamente, el aprendizaje móvil de lenguas (Mobile Assisted Language Learning, MALL de sus siglas en inglés). 
Son varias las ventajas señaladas en su uso. Entre ellas, destaca la observación, la negociación del significado, el aprendizaje mediante la práctica y la concentración en la forma (Doughty \& Long, 2003; Skehan, 2003). Además, las apps se muestran como herramientas idóneas para ofrecer oportunidades de participar en tareas interactivas, significativas y atractivas, fomentan tareas gratificantes y proporcionan oportunidades para realizar producciones en la lengua meta, tal y como defiende la teoría CALL de Oxford (1990), Chapelle (1998), Meskill (1999) y Skehan (2003).

Respecto al aprendizaje de lenguas, la investigación sostiene que para los usuarios estas herramientas de aprendizaje de lenguas resultan muy positivas (Brown et al., 2012; Khaddage \& Lattemann, 2013; Kim, 2013; Castañeda \& Cho, 2016), sobre todo en cuatro aspectos: la flexibilidad (Zou \& Li, 2015), la portabilidad, la capacidad para adecuar el aprendizaje de forma personalizada (Steel, 2012) y la autonomía (Chen, 2013). Tras haber realizado encuestas a los usuarios de dichas aplicaciones, parece que los estudiantes las utilizan de forma diferente a lo que sus creadores imaginan ya que solamente emplean una parte de las funcionalidades posibles.

Más en concreto, se ha confirmado la mejora en el aprendizaje en varias competencias lingüísticas como el vocabulario (Steel, 2012; Yildiz, 2012), la lectura y la escritura (Steel, 2012; Morgana \& Shrestha, 2018), la conciencia fonológica y la comprensión oral (Yildiz, 2012; Kim, 2013) así como en la conjugación verbal (Castañeda \& Cho, 2016).

Sin embargo, sus cualidades pedagógicas han sido puestas en duda, puesto que, entre otras carencias, la práctica lingüística parece poco equilibrada (Pareja-Lora et al., 2013), las actividades son básicas (Burston, 2014) y se centran en la cognición y la recepción sin trabajar de modo sistemático la producción. Las actividades de aprendizaje en las aplicaciones móviles se describen como elementales puesto que en su mayoría reproducen lo que se ha hecho antes con otras tecnologías. Se centran en procesos cognitivos y receptivos y carecen de oportunidades para llevar a cabo actividades sociocognitivas (Kim \& Kwon, 2012).

También aparecen contradicciones en la bibliografía consultada en lo que se refiere al aprendizaje colaborativo. En efecto, unos autores señalan que la utilización de las apps favorece dicho aprendizaje (Doughty \& Long, 2003; Skehan, 2003), mientras que otros indican que las oportunidades que aprendizaje colaborativo resultan más bien escasas cuando estas son utilizadas (Kim \& Kwon, 2012).

Claramente, las aplicaciones no son actualmente la solución única para el aprendizaje de lenguas, pero pueden apoyar eficazmente el aprendizaje, en aspectos como la autonomía y el interés por aprender una lengua (Goodwin-Jones, 2011).

Además, podemos considerar que estas herramientas pueden ser un buen complemento al aprendizaje formal y un óptimo punto de partida para los estudiantes de lenguas principiantes o estudiantes que desean mantener un nivel de lengua sin tener que seguir un aprendizaje formal (Rosell-Aguilar, 2017). Las investigaciones llevadas a cabo hasta el mo- 
mento se han centrado en la utilización dentro del aula, como complemento al aprendizaje formal, lo que ofrece resultados parciales, pero resultaría muy complejo desde el punto de vista metodológico y de selección de la muestra un estudio en ámbitos no formales de aprendizaje de lenguas.

Por lo tanto, en los estudios de las apps como instrumento de aprendizaje de una LE se señalan como ventajas aspectos como la autonomía, la flexibilidad para adaptarse al aprendizaje y la motivación. Además, se ha constatado un incremento en la competencia lingüística de los estudiantes. Sin embargo, los ejercicios se consideran repetitivos y no se llega a un consenso sobre el desarrollo del aprendizaje colaborativo.

Teniendo en cuenta lo anteriormente analizado en otros estudios, abordaremos a continuación el análisis de cuatro aplicaciones que permiten al usuario aprender FLE.

\subsection{Descripción e investigación previa de Duolingo, Busuu, Français Premiers Pas y Le Bon Mot}

En el ámbito del aprendizaje de FLE, existe una gran variedad de aplicaciones móviles: unas generalistas, esto es, aplicaciones que ofrecen la opción de aprender varias lenguas entre las que se encuentra el francés, y, otras específicas, es decir, aplicaciones desarrolladas únicamente para el aprendizaje de FLE. En este sentido, nos decantamos por analizar dos aplicaciones generalistas, Duolingo y Busuu, y dos específicas, Français Premiers Pas y Le Bon Mot. Esta decisión tiene como objetivo no solamente analizar los ejercicios y actividades propuestos por dos tipos de aplicaciones, sino también compararlos para determinar su eficacia en el aprendizaje de FLE y su utilidad a nivel comunicativo. Además, como veremos más adelante, a día de hoy existen estudios sobre Duolingo y Busuu, dos aplicaciones muy reconocidas por el público general, pero no se han analizado con tanta profundidad Français Premiers Pas y Le Bon Mot, dos aplicaciones desconocidas para el público general y escasamente conocidas entre el público interesado por aprender FLE. Aportamos así una visión contrastiva sobre las apps, incidiendo sobre dos de ellas, poco presentes en la bibliografía hasta ahora.

Así pues, para poder llevar a cabo dicho análisis, estudiaremos en primer lugar las características de estas cuatro aplicaciones. Empezaremos por las dos aplicaciones generalistas, Duolingo y Busuu, y después analizaremos los rasgos de las dos específicas, Français Premiers Pas y Le Bon Mot.

\subsubsection{Duolingo}

Duolingo es una aplicación interactiva creada en 2011 por Louis von Ahn y Severin Hacker. Esta app se descarga de forma gratuita, pero para acceder a contenidos mejorados se debe pagar una inscripción. Está disponible en las plataformas Android, iOS y Windows 
Phone. Según la lengua de la interfaz las opciones para el hispanohablante son las siguientes: inglés, francés, alemán, portugués, italiano, catalán, esperanto, guaraní, sueco y ruso. Pero las opciones se amplían mucho si la lengua de la interfaz es el inglés. También se añaden lenguas minoritarias como el finlandés o el navajo y lenguas artificiales como el Klingon.

Vesselinov y Grego (2012) afirman que aprender una lengua utilizando Duolingo durante 34 horas equivale a estudiar en la universidad durante 11 semanas o un semestre.

En una investigación con estudiantes principiantes de turco, Loewen et al. (2019) sugieren que los creadores de la app tendrían que introducir las teorías sobre el aprendizaje de lenguas en su desarrollo, dado que a menudo los ejercicios aparecen descontextualizados, pero sí que se advierte una mejora en los aprendices que la utilizaron más tiempo, así como un incremento en su motivación.

Munday (2015) realiza un estudio con alumnos de español como lengua extranjera. Tras una encuesta, lo más apreciado por ellos fue la sencillez de su uso, la facilidad de acceso a través de los dispositivos móviles (considerada como más atractiva que los libros). Otro aspecto que destacaron los estudiantes es la gamificación o el aspecto lúdico de esta herramienta en el aprendizaje de la LE (Schmoll, 2016). Además, un 10\% de los alumnos siguieron utilizando la app tras la finalización de las clases. Como aspectos negativos se señalan los fallos en la traducción. Esta autora considera Duolingo como un complemento válido de las clases formales puesto que, como resulta evidente, aumenta el tiempo de exposición de los alumnos a la lengua aprendida.

Respecto al ámbito específico del FLE y, más concretamente, respecto al vocabulario, Bradiyah et al. (2016) señalan que este es demasiado fácil incluso para estudiantes de nivel A1, y por ello concluyen que los niveles de vocabulario presentados en esta app se adecuarían a un nivel $\mathrm{A} 1$ junior.

Las investigaciones llevadas a cabo sobre la aplicación Duolingo evidencian que se produce una mejora en el aprendizaje de la lengua escogida (Vesselinov \& Grego, 2012) y que los estudiantes aprecian la simplicidad de su uso (Munday, 2015). También sugieren mejoras como incrementar la complejidad del vocabulario (Bradriyah et al., 2016) o contextualizar el aprendizaje (Loewen et al., 2019).

\subsubsection{Busuu}

La aplicación Busuu forma parte de la red Busuu para el aprendizaje de lenguas, que también incluye un sitio web. Fue creada en 2008 por Bernhard Niesner y Adrian Hilti.

Con más de 60 millones de usuarios registrados (Busuu, 2016), ofrece 12 lenguas diferentes organizados por niveles según el Marco Común Europeo de Referencia para las Lenguas (MCER, 2001): inglés, español, francés, alemán, japonés, italiano, ruso, chino, árabe, turco, portugués y polaco. La aplicación funciona en los sistemas operativos $A n-$ droid e iOS. Los alumnos tienen que registrarse para utilizarla y pueden elegir aprender 
una o más lenguas utilizando los materiales gratuitos o pasando a una suscripción premium de pago.

La investigación sobre el uso de Busuu es limitada, si bien se han realizado algunos estudios. En un estudio piloto, Kétyi (2013) encuestó a 59 estudiantes húngaros de alemán que recibieron una prueba gratuita de 7 días de membresía premium. El servicio tuvo una buena acogida, ya que el $79 \%$ de los participantes lo calificó de bueno o muy bueno. Los aspectos más beneficiosos identificados fueron el aprendizaje de vocabulario, el repaso y la práctica de la lengua, la facilidad y el carácter lúdico del aprendizaje, el uso de audio para la pronunciación y la selección de temas y vocabulario. Sin embargo, algunos participantes consideraron que las tareas eran demasiado fáciles y repetitivas. Tras la prueba gratuita, el $92 \%$ de los participantes indicó que no pagarían por la suscripción premium.

En un estudio de seguimiento, Kétyi (2015) comparó un grupo experimental de 51 estudiantes que utilizaban la versión premium de esta aplicación con un grupo de control de 43 estudiantes que no la utilizaban. Los usuarios de la aplicación se servían de ella aproximadamente una vez a la semana durante una media de entre 10 y 15 minutos. Más del 73\% de ellos consideraron que la aplicación era útil para el aprendizaje de lenguas sobre todo para el vocabulario y la práctica de la escritura.

Se apreció un aumento en la motivación en el grupo experimental y un incremento en la competencia lingüística de la lengua objeto, mientras que el grupo de control no lo hizo. A pesar de ello, la gran mayoría de los participantes manifestó que no pagarían por la aplicación después del periodo de prueba.

En otro estudio, Malerba (2015) indicó que los alumnos valoraban especialmente la flexibilidad de aprender a su propio ritmo, pero el número limitado de tipos de actividades era su principal razón para abandonar dichas plataformas de aprendizaje de lenguas.

En un estudio con 21 estudiantes de inglés como segunda lengua que utilizaban Busuu, Liu et al. (2013) descubrieron que el 52\% de los participantes la usaron fuera del aula durante más o menos 30 y 60 minutos por sesión. Las características favoritas de los estudiantes eran la interacción con los demás, el aprendizaje de nuevo vocabulario y la práctica de la lectura y la gramática. Los autores de este informe no especificaron si los estudiantes habían utilizado la versión de escritorio o la de aplicación. Abogaron por seguir investigando sobre las características específicas del sitio para determinar qué características de diseño tienen mayor potencial para los estudiantes de lenguas.

Vesselinov y Grego (2016) realizaron un estudio extensivo sobre Busuu. Este estudio hizo un seguimiento de las mejoras en el aprendizaje de español de 144 estudiantes autónomos que utilizaron Busuu durante dos meses, con pruebas previas y posteriores utilizadas como mediciones. Tras este periodo, el $84 \%$ de los participantes había mejorado su competencia escrita y el 75\% había mejorado su competencia oral. Los participantes más exitosos fueron los que invirtieron más tiempo en utilizar el servicio. Los factores como la edad, el 
sexo, el nivel educativo, el origen étnico, el dispositivo utilizado, la lengua materna o las razones para estudiar español no tuvieron un efecto estadísticamente significativo. El estudio también midió los niveles de satisfacción de los usuarios con resultados muy positivos. Los participantes utilizaron tanto la versión web (58\%) como la versión de la aplicación (42\%) del servicio, pero los autores del informe no proporcionaron un análisis de los resultados en función de la versión utilizada.

Rosell-Aguilar (2018) en una extensa encuesta sobre Busuu concluye que cuanto más tiempo se pasa con la app, más proclives a pagar por ella son los usuarios. Como en otros estudios, la manera de trabajar el vocabulario fue la más apreciada por los usuarios y el hecho de que los ejercicios sean repetitivos es lo menos valorado por los encuestados.

De todas estas investigaciones podemos deducir que una mayor exposición a la lengua mejora la adquisición de una LE y que todo el análisis de la eficacia de las apps en el aprendizaje de una LE, por ser dispositivos recientes y en cambio constante, requieren de un mayor estudio para mejorar sus prestaciones. Los usuarios encuestados, por su parte, valoran positivamente la facilidad de su uso y la motivación del aprendizaje. Destacan asimismo que favorecen la adquisición del vocabulario. Aun así, no muchos estarían dispuestos a pagar por la utilización de estas herramientas.

Tras analizar las aplicaciones generalistas, pasamos ahora a detallar las apps específicas en el aprendizaje del FLE.

\subsubsection{Français Premiers Pas}

Se trata de una aplicación creada en 2015 por el Cavilam (Vichy) y la fundación de la Alliance Française junto con la Universidad de Clermont Auvergne.

Ha sido concebida por profesores expertos en la didáctica del FLE: Robert Angéniol, Michel Boiron, Julie Miclo y Emmanuel Zimmert. Su descarga es gratuita y solamente se refiere al francés y a un nivel debutante. Así lo indica en su presentación: "une application ludique d'apprentissage du français pour débutants complets" (una aplicación lúdica de aprendizaje de francés para principiantes (CCavilam Alliance Française, 2020). Tras contactar con ellos por correo electrónico (marzo 2021), los editores de esta app nos han confirmado que no tienen constancia de que se haya realizado ningún estudio sobre la misma ${ }^{1}$.

\subsubsection{Le Bon Mot}

Por último, Le Bon Mot es una app creada en 2012 y dedicada exclusivamente al francés. Como Français Premiers Pas, ha sido diseñada por profesores especialistas de francés de una escuela francesa en Viena. Su efectividad fue contrastada por Chouchani (2013) que también formó parte del equipo desarrollador de la aplicación. Como productor ejecutivo

1 “À ma connaissance, aucune étude n`a été faite sur notre application Français premiers pas". Correo redactado por Emmanuel ZIMMERT y recibido el 19-03-21. 
figura Christian Mayr; como productor ejecutivo del contenido, Céline Juyou, y, como desarrolladores, Philipp Bilina y Daniel Zimmermann (Chouchani, 2013).

Chouchani (2013) realizó encuestas a los estudiantes de francés que habían utilizado la app tras su concepción. Los resultados de dicho cuestionario son bastante similares a los descritos más arriba para Busuu y Duolingo. Los alumnos encuestados juzgaron favorablemente la app considerándola como buena o muy buena y también la variedad de temas presentados contó con su aprobación. Además, subrayaron como característica positiva el haber mejorado el vocabulario gracias a las fotos e imágenes, pero lamentaron no haber podido repetir en voz alta las palabras aprendidas. Los ejercicios de gramática fueron considerados como fáciles. La mayoría de los usuarios de Le Bon Mot destacó su carácter lúdico y motivador.

Encontramos, por lo tanto, una disimetría en los estudios científicos de las apps que demuestren su validez como herramienta en el aprendizaje de una LE. Las generalistas que proponen varios idiomas como Busuи у Duolingo poseen desde su creación el respaldo de abundantes investigaciones que indican sus puntos fuertes y sugieren mejoras. Sin embargo, las específicas de FLE no han sido todavía investigadas con detalle.

\section{Análisis de la competencia oral}

Para un análisis completo de las apps, Rosell-Aguilar (2017) propone una taxonomía exhaustiva divida en cuatro grandes grupos: aprendizaje de lengua, pedagogía, experiencia de usuario y tecnología. Nos centraremos aquí únicamente en la competencia oral, más concretamente, en la comprensión y la producción. Hemos escogido esta competencia ya que resulta, a nuestro entender, la más compleja de desarrollar correctamente en un planteamiento de aprendizaje autónomo. Por ello, hemos decidido estudiar las distintas estrategias didácticas que proponen las aplicaciones seleccionadas para hacer avanzar al usuario en el oral. Dado que la app Français Premiers Pas se centra únicamente en usuarios de nivel inicial, hemos analizado las estrategias de expresión oral en las apps indicadas en los niveles iniciales (nivel A1).

Hemos dividido este análisis en dos partes: por un lado, estudiamos la comprensión oral y, por el otro, la expresión oral. Sin embargo, hemos de precisar que, para un aprendizaje correcto, todos estos aspectos deben relacionarse correctamente entre sí, no trabajarse de modo aislado, e incluir la fonética ya que resulta muy necesaria para poder trabajar ambas competencias orales. 


\subsection{Comprensión oral}

La comprensión oral es la competencia más desarrollada, tanto en las aplicaciones generalistas como las específicas. La tecnología actual permite que el sonido se reproduzca de modo sencillo, con lo cual la posibilidad de implementar esta competencia resulta asequible en las cuatro apps analizadas. Aun así, son las generalistas las que más tipos de ejercicios ofrecen a su público como lo muestra la siguiente tabla.

\begin{tabular}{lcccc}
\hline & Busuu & Duolingo & Le Bon Mot & Français Premiers Pas \\
\hline $\begin{array}{l}\text { Escuchar y seleccionar la imagen } \\
\text { correcta }\end{array}$ & $\sqrt{ }$ & $\sqrt{ }$ & $\sqrt{ }$ & $\sqrt{ }$ \\
\hline Escuchar y repetir una frase & $\sqrt{ }$ & $\sqrt{ }$ & $\mathrm{X}$ & $\mathrm{X}$ \\
\hline Escuchar una frase e identificarla & $\sqrt{ }$ & $\sqrt{ }$ & $\sqrt{ }$ & $\sqrt{ }$ \\
\hline Escuchar diálogos & $\sqrt{ }$ & $\mathrm{X}$ & $\mathrm{X}$ & $\sqrt{ }$ \\
\hline Ejercicios de completar & $\sqrt{ }$ & $\sqrt{ }$ & $\sqrt{ }$ & $\mathrm{X}$ \\
\hline Ejercicios verdadero/falso & $\sqrt{ }$ & $\sqrt{ }$ & $\mathrm{X}$ & $\mathrm{X}$ \\
\hline Traducción & $\sqrt{ }$ & $\sqrt{ }$ & $\sqrt{ }$ & $\mathrm{X}$ \\
\hline $\begin{array}{l}\text { Escuchar una frase y seleccionar } \\
\text { las palabras para escribir la frase }\end{array}$ & $\mathrm{X}$ & $\sqrt{ }$ & $\mathrm{X}$ & \\
\hline
\end{tabular}

Tabla 1: taxonomía de los ejercicios de comprensión oral.

Todos estos ejercicios combinan el sonido, la imagen y la lengua escrita, elementos que son muy empleados no solo en los ejercicios que trabajan las competencias orales, sino también en los que permiten al alumno desarrollar las competencias escritas, el vocabulario, la gramática o la sintaxis. Muchos de ellos, ofertados en su mayoría por Busuu y Duolingo, son tradicionalmente utilizados en el aprendizaje impartido por el docente en el aula: ejercicios de completar o indicar si una serie de afirmaciones son verdaderas o falsas, ejercicios de escuchar y repetir una frase, ejercicios de traducción o aquellos que se sirven de diálogos. Algunos también son compartidos por Le Bon Mot (ejercicios de completar o traducción) y, en menor medida, por Français Premiers Pas (escuchar diálogos). Pero debemos destacar que las cuatro aplicaciones coinciden en el uso de dos tipos de ejercicios: por un lado, los ejercicios en los que el estudiante debe escuchar y asociar lo escuchado con una imagen, $\mathrm{y}$, por el otro, aquellos en los que el alumno escucha una frase y debe escoger la respuesta correcta entre varias opciones.

Duolingo basa muchos de sus ejercicios en traducción directa (del francés al español) o inversa (del español al francés). Cada vez que el usuario selecciona una palabra esta se reproduce automáticamente en francés. Por ejemplo, tomemos la frase propuesta para la traducción: 
Anales de Filología Francesa, n. ${ }^{\circ}$ 29, 2021

ESTUDIO CONTRASTIVO DE LAS COMPETENCIAS ORALES EN LAS APLICACIONES MÓVILES (APPS)...

El café es malo.

Al elegir cada una de las palabras que constituyen la frase el sistema las articula de modo independiente, permitiendo así al usuario familiarizarse con la fonética francesa.

Sucede lo mismo cuando se trata de traducir del español al francés.

Elle marche jusque chez l'homme.

En ese caso, se activa inmediatamente el sonido y se puede oír la frase enunciada (2) palabra por palabra.

Otro ejercicio más específico de comprensión oral aparece enunciado como "escucha y selecciona las palabras". En la parte superior de la interfaz, emergen dos símbolos para escuchar: uno, con un micrófono y, otro, con una tortuga. El primero permite escuchar la frase a velocidad normal y, el segundo (el marcado con un icono de una tortuga), más despacio para adaptarse al ritmo del alumno. En la parte central, se realiza el ejercicio, en este caso, escribir la frase escuchada a partir de una serie de palabras que aparecen en la parte inferior de la interfaz (3).

Bonjour demain bienvenue va et tard d'accord

Estas palabras permiten a los alumnos construir la frase que acaban de escuchar, lo que les obliga a conocer la estructura de la frase simple. De manera que, además de desarrollar la competencia oral, se trabaja la sintaxis.

Como en el ejercicio anterior, se permite escuchar la frase propuesta tantas veces como se desee o se necesite e, incluso, se pueden escuchar las palabras por separado. Al ser pronunciadas palabra por palabra, aunque se puede conseguir una relación entre lo visual (la ortografía) y el escrito, no se trabaja ni la prosodia ni el ritmo de la frase.

Busuu, por su parte, permite la escucha y la lectura de diálogos situados en el contexto de la lección. Como en Duolingo, este ejercicio aúna la comprensión oral y escrita puesto que dichas conversaciones se acompañan de su transcripción escrita. En un segundo momento, el diálogo se vuelve a repetir, esta vez con huecos en cada una de las frases y el usuario debe completarlas con la palabra adecuada. Para ello, Busuu ofrece tres opciones y la posibilidad de escuchar otra vez cada una de las réplicas del diálogo para poder identificar correctamente cada uno de los términos y así completar dicho diálogo.

Anna: J'ai vraiment envie de voir des cultures différentes et des célèbres pour changer cette année.

a) Musées b) parc national c) monuments

James: D'accord, on pourrait faire une beaucoup de et J'adore les villes avec

a) Escapade en ville b) galeries c) musées. 
Para aprender el vocabulario, esta aplicación presenta una foto, la palabra escrita y una frase en la que aparece contextualizado el término. Tanto la palabra como la frase se puede escuchar y leer.

$$
\text { Confortable. J'adore ce pull, il est très confortable }
$$

Otro ejercicio propuesto por la aplicación Bussu es una pregunta cuya respuesta debe ser verdadero o falso. Se escucha una frase (6) que aparece escrita para facilitar la comprensión del alumno.

(6) Tu nous as préparé quoi de bon?

Y, basándose en lo que el alumno ha aprendido, se le plantea una pregunta sobre el uso de la frase (6).

¿Puedes hacer esta pregunta para averiguar qué ha cocinado tu amigo? Verdadero / Falso.

Como podemos comprobar, en estos ejercicios también se combina la comprensión oral y escrita.

Además de ejercicios que trabajan la comprensión oral y escrita, Busuu opta por otros que resultan muy adecuados para la comprensión oral.

En uno de ellos, se le pide al usuario que escoja la opción correcta. Por ejemplo, se escucha una frase como la siguiente (8):

(8) Tu veux que je t'aide? Non, c'est bon.

Y, asociada a dicha frase, se proponen tres opciones escritas en francés:

a) Marc n'a pas besoin d'aide pour mettre la table

b) Julie ne veut pas aider Marc à mettre la table

c) Marc veut de l'aide pour mettre la table.

Además, el usuario pude repetir la frase escuchada tantas veces como desee, lo que se adecúa a su ritmo de aprendizaje.

Lo mismo ocurre en otro ejercicio en el se le pide completar el espacio en blanco con ayuda del audio:

$$
\text { C'est sympa de nous avoir }
$$


El usuario debe escribir exactamente la palabra escuchada y no se dan opciones para completarla. Si no reproduce exactamente lo escuchado, el sistema repite el ejercicio.

Por último, se ofertan vídeos cortos (de no más de 60 segundos) y posteriormente se reproducen extractos de los mismos para que el estudiante pueda ir completando preguntas $\left(\mathrm{QCM}^{2} \mathrm{o}\right.$ abiertas) y verifique así la comprensión del discurso oral.

¿Qué hay en el bolso de Sophie?
a) Sus llaves, su monedero, su ordenador y su tarjeta bancaria
b) sus llaves, su monedero, su móvil y su tarjeta bancaria
c) sus llaves, su monedero, su móvil y su ordenador.

Tras el visionado del vídeo y mientras realiza el ejercicio de comprensión, el alumno puede consultar el vídeo.

Una vez contestado el test, aparece la transcripción en español/francés. En ocasiones, el vídeo aparece con subtítulos en francés para facilitar su comprensión.

Encontramos, por lo tanto, mayor número de ejercicios de comprensión en Busuu que cuidan la prosodia y permiten la comprensión de frases en contexto.

Le Bon Mot posee un fondo sonoro de canciones francesas que puede ser útil para captar la prosodia y el ritmo del francés. Asimismo, cuando el ejercicio solicitado es de ordenar una frase, el sistema la repite en francés en bloque, en vez de hacerlo palabra por palabra como el caso de Duolingo, lo que ayuda a acoger el ritmo de la frase y a realizar todas las liaisons correctas.

Berlin est en Allemagne [Berlẽctãləman] Je suis à Munich [3əsuiza*mynik]

Estos ejercicios permiten al alumno, desde un nivel inicial, advertir les liaisons en [t] (12) y en [z] (13). E incluso a adquirir la entonación descendente de la frase interrogativa, como en (14).

De quelle nationalité elles sont?

Además, los ejercicios propuestos para trabajar la comprensión oral tienen como ejes el sonido y el lenguaje escrito, pero incorporan imágenes directamente relacionadas con el contenido de los ejercicios que facilitan su realización. Tanto en los ejercicios que el alumno debe seleccionar la respuesta correcta entre varias opciones como en los que se debe ordenar una frase, el sistema señala en rojo la respuesta incorrecta y la correcta en verde, pero solo repite en francés la respuesta correcta.

2 Las siglas QCM o Questions à Choix Multiple en francés se refieren a las preguntas de opción múltiple. 
En esta aplicación, no hemos encontrado ejercicios específicos de comprensión oral más allá de la reproducción de los sonidos que integran los ejercicios propuestos.

Français Premiers Pas difiere del resto de aplicaciones. Esta app se basa en conversaciones que se producen en diversas situaciones de la vida cotidiana (en el hotel, en el restaurante o en la tienda de ropa) para enseñar francés. La aplicación permite trabajar las fórmulas que más se utilizan para reservar una habitación, pedir los platos en un restaurante o solicitar una prenda concreta. Este método se vale de la comprensión oral tanto para enseñar vocabulario, como para evaluar el aprendizaje. En todas las situaciones propuestas, se escucha una frase y se ofrecen unos dibujos con los que se debe identificar la frase. Este mismo ejercicio también se plantea a la inversa: se dan dos, tres o cuatro frases sonoras y hay que escoger la que corresponde al dibujo.

En la secuencia de ejercicios ambientados en el restaurante, por ejemplo, se emplea la conversación que mantienen un camarero y un cliente. En los primeros ejercicios, se trabaja la frase que el camarero dice al cliente:

Vous avez choisi?

El alumno debe asociar la frase a una de las dos imágenes que se le ofrecen: en la primera, el cliente está a la mesa y el camarero se va y, en la segunda, el camarero está mirando al cliente. En el siguiente ejercicio, en cambio, la fórmula del camarero se trabaja al revés: se ofrece la imagen en la que el camarero y el cliente mantienen la conversación, pero las opciones son dos frases sonoras. Si el alumno quiere hacer el ejercicio, debe pulsar el botón que contiene el icono de micrófono para poder oír la(s) frase(s). Una vez realizada esta primera intervención, el alumno trabaja la respuesta del cliente a dicha pregunta mediante ejercicios que también combinan frases sonoras e imágenes. En este caso, el uso de imágenes y frases sonoras permite aprender el nombre de los platos:

(16) Une salade/du poisson/une omelette/du poulet

En lo que ese conoce como entraînement, es decir, la etapa previa a la realización de los ejercicios, el alumno realiza otras actividades en las que adquiere el vocabulario necesario. Para ello, la aplicación emplea imágenes asociadas a los audios.

A medida que se van realizando ejercicios, el número de opciones pasa de dos a tres y luego a cuatro, incrementando la dificultad para el alumno. Así pues, Français Premiers Pas basa todo su sistema de aprendizaje y de evaluación de los ejercicios en el lenguaje oral. Como sucede con Le Bon Mot, permite activar los subtítulos para favorecer la comprensión.

A nivel fonético, la combinación de sonido y lengua escrita o las imágenes que hacen las aplicaciones permite al alumno familiarizarse tanto con los sonidos consonánticos como 
los vocálicos del sistema fonético del francés. Aunque, lo cierto es que estos ejercicios no trabajan específicamente la fonética.

Por lo tanto, Français Premiers Pas basa en la comprensión oral todo su sistema a través de frases que responden a cada una de las situaciones estudiadas.

En resumen, podemos decir que la comprensión oral se halla muy desarrollada en las apps aunque la oferta de ejercicios es muy dispar. En cuanto al tipo de ejercicios, a pesar de las facilidades que ofrece la tecnología para concebirlos, las generalistas son las más completas y las que más variedad ofrecen para trabajar esta competencia. Tanto las generalistas como las específicas basan el aprendizaje en la comprensión de la lengua oral lo que permite familiarizarse con los sonidos de la lengua (consonantes y vocales), aunque estos no son trabajados de manera sistematizada.

\subsection{Expresión oral}

Al igual que en los ejercicios anteriores, en los que se trabaja la comprensión oral, los ejercicios de expresión oral integran el sonido y el lenguaje escrito. Pero, a diferencia de aquellos, estos se centran en acciones como la repetición, la lectura o la grabación, muy necesarias para implementar la expresión oral, ya que el alumnado suele tener dificultades para expresarse oralmente en la lengua extranjera. En la tabla 2 resumimos los ejercicios de expresión oral que aparecen en las apps.

\begin{tabular}{lcccc}
\hline & Busuu & Duolingo & Le Bon Mot & Français Premiers Pas \\
\hline Repetir una frase & $\sqrt{ }$ & $\sqrt{ }$ & $\mathrm{X}$ & $\mathrm{X}$ \\
\hline $\begin{array}{l}\text { Leer una frase después de haberla } \\
\text { formado }\end{array}$ & $\mathrm{X}$ & $\sqrt{ }$ & $\sqrt{ }$ & $\mathrm{X}$ \\
\hline $\begin{array}{l}\text { Asociar frases para verificar la } \\
\text { comprensión }\end{array}$ & $\mathrm{X}$ & $\sqrt{ }$ & $\sqrt{ }$ & $\mathrm{X}$ \\
\hline Grabar un monólogo & $\sqrt{ }$ & $\mathrm{X}$ & $\mathrm{X}$ & $\mathrm{X}$ \\
\hline
\end{tabular}

Tabla 2: taxonomía de los ejercicios de expresión oral.

Duolingo permite escuchar una frase (17), grabarla y volver a escuchar la expresión del usuario.

$$
\text { C'est un mariage. }
$$

El sistema, además, posibilita la comparación entre la frase original y la formulada por el estudiante, también anima a la repetición de la palabra complicada: mariage. Sobre todo, es la consonante constrictiva sonora [3] la que resulta de una gran dificultad articulatoria para el público en general, y para el público hispanófono en especial, por tratarse de 
una consonante que no existe en el sistema fonético del español. Sin embargo, aunque la frase pronunciada quede lejos de una óptima pronunciación, el estudiante recibe siempre una evaluación positiva de su expresión oral. Este ejercicio nos recuerda a los laboratorios de idiomas, que en sus comienzos resultaron revolucionarios, porque permitían al estudiante escuchar su voz. La tecnología actual permite este ejercicio con instrumentos tecnológicos al alcance de muchos.

Este aspecto se corrige en Busuu. En esta aplicación, si el usuario realiza una expresión oral con una pronunciación deficiente, el sistema envía un mensaje en rojo, lo que significa una evaluación negativa e incita al estudiante a repetir la frase de nuevo ("prueba otra vez").

Esta aplicación incorpora, además, lecciones específicas sobre fonética en las que se trabajan la articulación de consonantes o vocales para que el alumno mejore a nivel articulatorio, un aspecto necesario para una correcta expresión oral. Un ejemplo de ello lo encontramos en la lección 7 del nivel A1, en la se trabaja los sonidos [y] et [u]. Como el público en general no suele tener una formación fonética, emplean las grafías asociadas a las dos vocales, es decir, u y ou respectivamente. Estas lecciones son muy completas, ya que, por un lado, se lleva a cabo una explicación de su articulación mediante vídeos cortos en los que el alumno puede escuchar palabras que han aprendido en lecciones anteriores y que contienen los sonidos. Para $[\mathrm{u}]$ emplean las siguientes palabras:

Bonjour, tout, vous y roue

Y para $[y]$ estas otras:

Salut, tu, vue y rue

Las indicaciones escritas se completan con vídeos en los que una persona muestra la posición de la boca para poder pronunciar correctamente. Asimismo, se emplean ejercicios de discriminación de sonidos para aprender a distinguir ambos sonidos, lo que permite pronunciarlos fácilmente.

Otra característica interesante de esta app es la posibilidad de grabar una frase o un monólogo como respuesta a la intervención de un locutor en una situación de la vida cotidiana. Dicho ejercicio se lleva a cabo al final de cada lección y la grabación es enviada para su corrección a un usuario que forma parte de la comunidad creada por la propia aplicación. Este usuario tiene como lengua materna la lengua estudiada. Por ejemplo, al final de la lección 3, llamada “Comment ça va?", el alumno debe contestar oralmente o por escrito a la pregunta:

$$
\text { Comment te sens-tu aujourd'hui? }
$$


(20) permite utilizar las frases y el vocabulario trabajados para expresar las sensaciones del momento. Una vez el ejercicio completado, la aplicación envía este ejercicio a un usuario nativo para que lo corrija. Así, se puede crear una comunidad virtual de intercambio de idiomas y los alumnos pueden recibir la corrección, y comprobar de este modo si están adquiriendo la lengua correctamente.

Le Bon Mot propone ejercicios en los que se trabaja la estructura de la oración y la lectura posterior de las mismas para trabajar la articulación y aspectos prosódicos como el ritmo. Pero no ofrece la oportunidad de grabar lo leído ni cotejarlo con una corrección propuesta por la aplicación o de corregir mínimamente el ejercicio como hace Busuu.

Respecto a Français Premiers Pas, no existen ejercicios o actividades específicos que trabajen la expresión oral. Advertimos que el uso de ejercicios contextualizados permite al alumno aprender fórmulas para realizar diferentes actos de habla (presentarse, pedir, reservar...) pero sin poderlas poner en práctica por falta de ejercicios que permitan la expresión oral, aunque el usuario podría realizar este ejercicio de modo autónomo.

Por lo tanto, Busuu es la aplicación que destaca en la oferta de posibilidades para trabajar la expresión oral y la fonética, seguida por Duolingo. Las aplicaciones específicas no trabajan esta competencia o, como Français Premiers Pas, carecen de ejercicios que lo hagan a pesar de que los alumnos aprenden fórmulas que les pueden ayudar a llevar a cabo una conversación muy sencilla en contextos reales de comunicación verbal.

\section{Conclusión}

Tras analizar los ejercicios que permiten poner en práctica las competencias orales, basándonos en trabajos que estudian específicamente Duolingo y Busuu, asumimos que las apps las trabajan de manera desigual.

La comprensión oral, gracias a la tecnología actual, aparece mucho más desarrollada en las apps aunque la oferta de ejercicios es muy dispar. Las generalistas (Duolingo y Busuu) presentan mayor número de ejercicios y muy diferentes, pero estos solo dan por válida una de las respuestas posibles. Respecto a las apps específicas de francés destaca Français Premiers Pas que basa sus principios didácticos en asociar imágenes y frases relacionadas con las situaciones de comunicación. Le Bon Mot enuncia frases completas permitiendo así al usuario familiarizarse con la prosodia del francés.

Respecto a la expresión oral, las apps específicas de FLE no dan la posibilidad al usuario de expresarse oralmente. Duolingo y Busuu, en cambio, sí que lo hacen, aunque con ciertas diferencias: la primera presenta deficiencias en la corrección de las producciones reales, y la segunda es, a nuestro entender, la app que mejor desarrolla este aspecto porque, además, crea una comunidad de hablantes nativos para corregir la expresión oral.

Por último, debemos señalar que un análisis completo de las apps incluiría muchos 
más aspectos (Rosell-Aguilar, 2017) que no podemos abarcar en esta contribución pero que serán objeto de ulteriores investigaciones. Del mismo modo, nos parecen altamente pertinentes las encuestas a los usuarios que han demostrado su fiabilidad para evaluar el potencial didáctico de las diferentes apps. Por lo tanto, utilizaremos cuestionarios para profundizar en el estudio de las apps específicas de FLE.

\section{Referencias bibliográficas}

Bradiyah, Rima, Yuliarti Mutiarsih \& Dante Darmawangsa. 2016. "Analyse des items du test de vocabulaire français multimédia interactif Duolingo" in Actes de la Conférence Internationale sur le Français (CIF) 2016 "Le français: enjeux linguistiques, politiques, économiques, et culturels, 38-48.

Brown, Marnie, Joachim Castellano, Erin Hughes \& Alexander Worth. 2012. "Integration of iPads into a Japanese University English Language Curriculum" in JALT CALL Journal, nº 8(3), 197-209.

Burston, Jack. 2014. "The Reality of MALL: Still on the Fringes" in CALICO Journal, $\mathrm{n}^{\circ}$ 31(1), 103-125.

BusuU: $<$ https://www.busuu.com/es> [08/07/2021].

Castañeda, Daniel \& Cho, Moon-Heum. 2016. "Use of a game-like application on a mobile device to improve accuracy in conjugating Spanish verbs" in Computer assisted language learning, $\mathrm{n}^{\mathrm{o}}$ 29(7), 1195-1204.

Chapelle, Carol. 1998. "Multimedia CALL: Lessons to Be Learned from Research on Instructed SLA" in Language learning and Technology, vol. 2, $\mathrm{n}^{\circ}$ 1, 22-34.

CHEN, Xiao-Bin. 2013. "Tablets for informal language learning: Student usage and attitudes" in Language Learning \& Technology, $\mathrm{n}^{\circ}$ 7, 20-36.

Chouchani, Johanna. 2013. Les relations entre méthodologie des langues et enseignement/ apprentissage su français sur smarthphone: entre stimulations et contraintes. Mémoire de Master.

Conseil De L'EuRope. 2001. Cadre européen commun de référence pour les langues:appre ndre,enseigner,évaluer. Paris, Didier. < http://www.coe.int/t/dg4/linguistic/source/framework fr.pdf $>$ [08/07/2021].

CONSEIL De L'Europe. 2018. Cadre européen commun de référence pour les langues: apprendre, enseigner, évaluer. Volume complémentaire avec de nouveaux descripteurs: <www. coe.int/lang-cecr $>[08 / 07 / 2021]$.

Doughty, Catherine \& Michael, Long. 2003. "Optimal Psycholinguistic Environments for Distance FL Learning" in Language Learning and Technology, vol. 7, n 3, 50-80.

DuOLINGO: $<$ https://es.duolingo.com $>$ [08/07/2021].

FrANÇAIS PREMIERS PAS: <https://www.leplaisirdapprendre.com/portfolio/pas-a-pas/> [08/07/2021]. 
Anales de Filología Francesa, n. ${ }^{\circ}$ 29, 2021

ESTUDIO CONTRASTIVO DE LAS COMPETENCIAS ORALES EN LAS APLICACIONES MÓVILES (APPS)...

Godwin-Jones, Robert. 2011a. "Emerging Technologies: mobile apps for language learning” in Language Learning \& Technology, vol. 15, $\mathrm{n}^{\circ}$ 2, 2-11.

KÉTYI, András. 2013. "Using Smart Phones in Language Learning - A pilot study to turn CALL into MALL" in Bradley, Linda \& Sylvie Thouësny (eds.). 20 Years of EUROCALL: Learning from the Past, Looking to the Future. Proceedings of the 2013 EUROCALL Conference, Evora, Portugal. Dublin, Ireland: Research-publishing.net, 129-134.

KÉTYI, András. 2015. "Practical evaluation of a mobile language learning tool in higher education”. In Francesca Helm, Linda Bradley, Marta Guarda, \& Sylvie Thouësny(eds.), Critical CALL - Proceedings of the 2015 EUROCALL Conference, Padova, Italy. Dublin,Ireland:Researchpublishing.net,306-311. <http://dx.doi.org/10.14705/rpnet.2015.000350> [08/07/2021].

KIM, Heyoung \& Yeonhee KwON. 2012. "Exploring smartphone applications for effective mobile-assisted language learning" in Multimedia-Assisted Language Learning, $\mathrm{n}^{\mathrm{o}}$ 15(1), 31-57.

Le Bon Мот: <https:/www.lebonmot.app/es/> [08/07/2021].

Liu, Min; Evans, Matthew; Horwitz, Elaine; Lee, Sunjung; Mccrory, Monica; Park Jeong-Bin \& Claire Meadows Parrish. 2013. "A study of the use of social networking sites for language learning by university ESL students" in Lamy, Marie-Noëlle \& Katherine Zorou (ed.). Social networking for language education. Basingstoke, UK, Palgrave Macmillan, $15-41$.

Loewen, Shawn; Crowther, Dustin; Isbell, Daniel R.; Kim, Kathy M; Maloney, Jeffrey; Miller, Zachary \& Hima Rawal. 2019. "Mobile-assisted language learning: A Duolingo case study" in ReCALL (Cambridge, England, 31(3), 293-311. g: A Duolingo Case Study." Recall 31:3 (September 2019), 293-311: <https://doi.org/10.1017/S0958344019000065> [08/07/2021].

Malerba, María Luisa. 2015. Learners'behaviours and autonomy in LiveMocha and busuu online communities. Paper presented at de EDEN 2015 annual conference, Barcelona, Spain, $11^{\text {th }}$ June.

Meskill, Carla 1999. "Computers as Tools for Sociocollaborative Language Learning” in K. Cameron (ed.). CALL: Media, Design and Applications, (141-162). Lisse: Swets \& Zeitlinger.

Morgana, Valentina \& Prithvi Shrestha. 2018. “Investigating Students' and Teachers' Perceptions of Using the iPad in an Italian English as a Foreign Language Classroom" in International journal of computer-assisted language learning and teaching, vol. 8, $\mathrm{n}^{\circ}$ 3, 29-49.

Munday, Pilar. 2015. "The case for using Duolingo as part of the language classroom experience" in Revista iberoamericana de educación a distancia, vol. 19, nº1, 83-101.

Pareja-Lora, Antonio; Arús-Hita, Jorge; Martín Monje, Elena; Read, Timothy; Pomposo-Yanes, Lourdes; Rodríguez-Arancón, Pilar; Calle-Martínez, Cristina \& Elena BÁRCENA. 2013. Toward Mobile Assisted Language Learning apps for professionals that integrate learning into the daily routine in BRADLEY, Linda \& Sylvie THOUËSNY (eds.). 20 Years of EUROCALL: Learning, from the Past, Looking to the Future. Proceedings of the 2013 EUROCALL Conference, Evora, Portugal. Dublin, Ireland, 206-210. 
Rosell-Aguilar, Fernando. 2017. "State of the App" in CALICO Journal, vol. 34, n², 243-258.

Rosell-Aguilar, Fernando. 2018. “Autonomous language learning through a mobile application: a user evaluation of the busuu app" in Computer Assisted Language Learning, vol. $31, n^{\circ} 8,854-881$.

SchmolL, Laurence. 2016. "L'emploi des jeux dans l'enseignement des langues étrangères: Du traditionnel au numérique" in Sciences du jeu, 5: <http://journals.openedition.org/sdj/628; DOI: $10.4000 /$ sdj.628> [08/07/2021].

Skehan, Peter. 2003. "Focus on Form, Tasks, and Technology" in Computer assisted language learning, vol. 16, $\mathrm{n}^{\circ} 5,391-411$.

SteEL, Caroline. 2012. Fitting learning into life: language students'perspectives on benefits of using mobile apps. ASCILITE.

Vesselinov, Roumen \& John Grego. 2012. Duolingo effectiveness study. City University of New York, USA, 1-25.

Vesselinov, Roumen \& John Grego. 2016. The busuu efficacy study: <http://comparelanguageapps.com/documentation/The_busuu_Study2016>[08/07/2021].

YILDIZ, Senem. 2012. Use of iPad applications to introduce English as a Foreign language to young Turkish learners. Paper presented at the 2012 CALICO Conference, University of Notre Dame, South Bend, IN. 
